\title{
CIENCIAMATRIA
}

Revista Interdisciplinaria de Humanidades, Educación, Ciencia y Tecnología

Año VIII. Vol. VIII. Nro 1. Edición Especial. 2022

Hecho el depósito de ley: pp201602FA4721

ISSN-L: 2542-3029; ISSN: 2610-802X

Universidad Nacional Experimental Francisco de Miranda (UNEFM). Santa Ana de Coro. Venezuela

Adriana Doménica Macías-Díaz; Erika Anabel Vivas-Pazmiño; Kenia Lisbeth Alvarado-Cuenca;

Luzmila Josefa Andrade-López

DOI 10.35381/cm.v8i1.665

\section{Globalización y derechos humanos}

\section{Globalization and human rights}

\author{
Adriana Doménica Macías-Díaz \\ dq.adrianadmd39@uniandes.edu.ec \\ Universidad Regional Autónoma de los Andes, Quevedo \\ Ecuador \\ https://orcid.org/0000-0003-0563-139X \\ Erika Anabel Vivas-Pazmiño \\ dq.erikavp51@uniandes.edu.ec \\ Universidad Regional Autónoma de los Andes, Quevedo \\ Ecuador \\ https://orcid.org/0000-0002-5617-8372 \\ Kenia Lisbeth Alvarado-Cuenca \\ dq.keilac36@uniandes.edu.ec \\ Universidad Regional Autónoma de los Andes, Quevedo \\ Ecuador \\ https://orcid.org/0000-0003-1953-3846 \\ Luzmila Josefa Andrade-López \\ dq.luzmilajal11@uniandes.edu.ec \\ Universidad Regional Autónoma de los Andes, Quevedo \\ Ecuador \\ https://orcid.org/0000-0002-1564-0938
}

Recibido: 15 de septiembre 2021

Revisado: 10 de noviembre 2021

Aprobado: 15 de diciembre 2021

Publicado: 01 de enero de 2022 


\section{CIENCIAMATRIA \\ Revista Interdisciplinaria de Humanidades, Educación, Ciencia y Tecnología \\ Año VIII. Vol. VIII. Nro 1. Edición Especial. 2022 \\ Hecho el depósito de ley: pp201602FA4721 \\ ISSN-L: 2542-3029; ISSN: 2610-802X \\ Universidad Nacional Experimental Francisco de Miranda (UNEFM). Santa Ana de Coro. Venezuela}

Adriana Doménica Macías-Díaz; Erika Anabel Vivas-Pazmiño; Kenia Lisbeth Alvarado-Cuenca; Luzmila Josefa Andrade-López

\section{Estimado Editor (a):}

Sin duda alguna la globalización de modo general ha provocado un gran impacto en los ámbitos social, político, económico, familiar y ambiental, en este sentido al abordar el tema globalización y derechos humanos, es necesario mirar los diferentes contextos que rodean dicho término, ya que para algunos, simboliza un progreso significativo en la mejora de los pueblos, pero para otros representa lo contrario. Para Moreno Arvelo, et al. (2021):

Los efectos de la globalización ante derechos humanos han llegado hasta tratados universales y es el resultado globalizador en este ámbito, ya que vinculan a los Estados partes por ser quienes protegen los sistemas regionales el cual es un fenómeno homogéneo (Trejo, 2017). Es decir, en el que involucran a todos de la misma manera; hasta la inclusión de protección de derechos y una nueva visión como son los derechos a la paz, la seguridad y protección del medio ambiente; los mismos que son aspectos positivos. (p.553)

En la práctica la realidad es diferente, ya que no todos los países han logrado cumplir con los requerimientos fundamentales de sus ciudadanos, vulnerando los derechos humanos por las fallas de su gestión gubernamental en donde los modelos de gobierno han fracaso, por ello Ciuro Caldani (2019) plantea:

La internacionalidad y la globalización/marginación pueden brindar ventajas inmediatas, a menudo particularizadas, pero no están en condiciones de logar de maneras satisfactorias el incremento sólido de la productividad, la inclusión y el fortalecimiento de las instituciones y la gobernanza que la integración y la comunitarización están en mejores condiciones de realizar. Por estas vías es mucho más posible la realización del ideal del desarrollo humano sustentable. (p.310)

La participación de la colectividad con sus respectivos gobiernos debería ser la respuesta, para disminuir las diferencias en materia de los derechos humanos, en este orden de ideas, mientras exista una sola comunidad en el mundo que no disfruta de 


\section{CIENCIAMATRIA}

Revista Interdisciplinaria de Humanidades, Educación, Ciencia y Tecnología

Año VIII. Vol. VIII. Nro 1. Edición Especial. 2022

Hecho el depósito de ley: pp201602FA4721

ISSN-L: 2542-3029; ISSN: 2610-802X

Universidad Nacional Experimental Francisco de Miranda (UNEFM). Santa Ana de Coro. Venezuela

Adriana Doménica Macías-Díaz; Erika Anabel Vivas-Pazmiño; Kenia Lisbeth Alvarado-Cuenca; Luzmila Josefa Andrade-López

todos los derechos humanos, la ética del desarrollo tendrá algo que decir al respecto, tanto para denunciar las injusticias como para "ayudar". (Martínez Navarro, 2017, p. 48) Es decir, las desigualdades se evidencian a nivel global, olvidando al ser humano como el centro de todo desarrollo, priorizando las riquezas económicas, lo que transgrede sus derechos fundamentales establecidos en las convenciones internacionales y suscritas por los diferentes países del mundo, en este orden de ideas Santano (2019) indica:

La pobreza, falta de oportunidades, desigualdades, entre otras cuestiones, no solo son muy comunes porque muchos gobiernos son ineficientes para alcanzar sus objetivos, sino también porque este fenómeno internacional facilitó el crecimiento de las desigualdades y la explotación de trabajadores. (p.43)

Desde esta visión, globalización y derechos humanos es antagónica, ya que no se complementa precisamente por el hecho de la falta de acciones que permitan un equilibrio y distribución equitativa de los recursos producto de rentas y ganancias por ello, Balbín (2003) realiza la siguiente aseveración:

Actualmente los estrategas de la globalización y los Movimientos de defensa de los Derechos Humanos, es decir, los Movimientos Sociales (y no sólo las organizaciones de DD.HH.), se sitúan en abierta oposición, unos frente a otros, a causa de disímiles y opuestas percepciones y valoraciones, tanto del proceso de globalización en curso, como de los Derechos Humanos como tales.(p.18)

Dichas aseveraciones, siguen vigentes a pesar de los años y que se han incrementado en los últimos tiempos producto de las malas acciones de los gobernantes, el Estado es quien debe cumplir con las tareas de coordinación, supervisión, regulación y control de todas las áreas relacionadas con la protección y desarrollo de sus ciudadanos. Sin embargo, Carrillo Punina (2017) plantea la globalización, como respuesta a una necesidad: 


\section{CIENCIAMATRIA}

Revista Interdisciplinaria de Humanidades, Educación, Ciencia y Tecnología

Año VIII. Vol. VIII. Nro 1. Edición Especial. 2022

Hecho el depósito de ley: pp201602FA4721

ISSN-L: 2542-3029; ISSN: 2610-802X

Universidad Nacional Experimental Francisco de Miranda (UNEFM). Santa Ana de Coro. Venezuela

Adriana Doménica Macías-Díaz; Erika Anabel Vivas-Pazmiño; Kenia Lisbeth Alvarado-Cuenca; Luzmila Josefa Andrade-López

La globalización surge como una necesidad del hombre que busca el desarrollo y progreso tanto económico como social y la tecnología, comunicación e información se han convertido en la fuente del progreso de las naciones y el abanico de oportunidades para crecer. (p.280)

Desde esta perspectiva como fuente de progreso se deben dar grandes avances en el desarrollo económico y social, disminuyendo las consecuencias desfavorables en materia de desigualdad, vinculando la globalización y los derechos humanos como políticas para la promoción de las buenas políticas económicas, sociales, ambientales, de salud y vida para la humanidad. Las comunidades, están llamadas a establecer alternativas como cambios medulares que deben estar integrados, promocionando nuevos paradigmas a nivel global desde el desarrollo de los derechos humanos.

\section{FINANCIAMIENTO}

No monetario.

\section{AGRADECIMIENTO}

A la Universidad Regional Autónoma de los Andes, Quevedo, por motivar el desarrollo de la Investigación.

\section{REFERENCIAS CONSULTADAS}

Balbín A., J. (2003) Globalización de los derechos... Repensando la condición humana: debates en derechos humanos. [Globalization of rights... Rethinking the Human Condition: Debates on Human Rights] Red de Bibliotecas Virtuales de CLACSO. Recuperado de: https://n9.cl/rluny

Carrillo Punina, Á. (2017) Globalización: revolución industrial y sociedad de la información. [Globalization: industrial revolution and information society.] Revista CIENCIA, 19, (2), 269-284. Recuperado de: https://n9.cl/vobpf 


\section{CIENCIAMATRIA}

Revista Interdisciplinaria de Humanidades, Educación, Ciencia y Tecnología

Año VIII. Vol. VIII. Nro 1. Edición Especial. 2022

Hecho el depósito de ley: pp201602FA4721

ISSN-L: 2542-3029; ISSN: 2610-802X

Universidad Nacional Experimental Francisco de Miranda (UNEFM). Santa Ana de Coro. Venezuela

Adriana Doménica Macías-Díaz; Erika Anabel Vivas-Pazmiño; Kenia Lisbeth Alvarado-Cuenca;

Luzmila Josefa Andrade-López

Ciuro-Caldani, M. (2019). Los procesos de integración frente a la crisis de gobernanza: perspectiva jurídica. [Integration processes in the face of the governance crisis: a legal perspective] Revista de la Secretaría del Tribunal Permanente de Revisión, 7(13), 307-314. https://dx.doi.org/10.16890/rstpr.a7.n13.p307

Martínez Navarro, Emilio. (2017). Ética del desarrollo en un mundo globalizado. [Ethics of development in a globalized world.] Veritas, (37), 35-50. https://dx.doi.org/10.4067/S0718-92732017000200035

Moreno Arvelo, P., Jaya Granados, N., Albán Valencia, J., y Zambrano Zambrano, A.(2021). Impacto de la globalización en la protección de los derechos humanos en Ecuador. [Impact of globalization on the protection of human rights in Ecuador] Revista Universidad y Sociedad, 13(5), 551-556. Recuperado de: https://n9.cl/yy7u0

Santano, A. (2019) Derechos humanos para el desarrollo de una sociedad realmente globalizada. [Human rights for the development of a truly globalized society] Opinión Jurídica, 19(38),39-57. https://doi.org/10.22395/ojum.v19n38a2

(C2022 por los autores. Este artículo es de acceso abierto y distribuido según los términos y condiciones de la licencia Creative Commons Atribución-NoComercial-Compartirlgual 4.0 Internacional (CC BY-NC-SA 4.0) (https://creativecommons.org/licenses/by-nc-sa/4.0/). 